\title{
An Adult Case of Thiamine-Sensitive Megaloblastic Anemia Syndrome Accidentally Diagnosed Myelodysplastic Syndrome
}

\author{
Ali Kutlucan
}

\begin{abstract}
Thiamine-responsive megaloblastic anemia (TRMA) syndrome is a rare disease comprising a classic triad of megaloblastic anemia, diabetes mellitus, and early-onset sensorineural deafness. TRMA can generally be diagnosed in early childhood. Early diagnosis is important to prevent complications that may develop soon. As it is a rare disease, diagnosis may sometimes be difficult. We present a rare case of an adult patient with TRMA who had been mistakenly diagnosed with myelodysplastic syndrome (MDS), whose anemia was corrected only after thiamine treatment was started.
\end{abstract}

Key Words: Thiamine-responsive megaloblastic anemia, Myelodysplastic syndrome, Thiamine, Autoimmune hypothyroidism.

\section{INTRODUCTION}

Thiamine-responsive megaloblastic anemia (TRMA) syndrome is a rare disease that was first described by Porter and colleagues, which demonstrates autosomal recessive $(A R)$ transmission and comprises the classic triad of megaloblastic anemia, diabetes mellitus (DM), and early-onset sensorineural deafness. ${ }^{1}$ It has been reported that these complications may be accompanied by findings such as cardiomyopathy, congenital heart defect, situs inversus, arrhythmias, short stature, developmental delay, ataxia, thrombocytopenia, pancytopenia, optic atrophy, retinal degeneration, and stroke.2,3 It has been reported that mutations in the SLC19A2 gene, which encodes the high-affinity thiamine trans-porter, result in TRMA. ${ }^{4}$ As a result of this mutation, thiamine deficiency occurs in affected tissues. So, cellular metabolism is impaired.2

Megaloblastic anemia usually begins early in life, during infant and adolescent periods when hyperglycemia occurs.5,6 Progressive hearing loss is irreversible and occurs very early. ${ }^{7}$

We present the case of a patient with TRMA in a 32-year woman diagnosed with anemia and myelodysplastic syndrome (MDS) for an extended period of time and whose anemia could not be corrected with MDS treatment but completely resolved in 4 months with thiamine treatment. Besides the classic triad, she had autoimmune hypothyroidism and obvious cardiac failure, which partially regressed with thiamine treatment. To our knowledge, such a phenomenon has not been reported previously in the literature.

Department of Hematology, Faculty of Medicine, Baskent University, Konya, Turkey

Correspondence: Dr. Ali Kutlucan, Department of Hematology,

Faculty of Medicine, Baskent University, Konya, Turkey

E-mail:dralikutlucan@gmail.com

Received: November 22, 2018; Accepted: February 06, 2019

\section{CASE REPORT}

A 32-year female patient was admitted to our clinic with complaints of fatigue and abdominal distension. The mental status of the patient was good. Due to her hearing problem, she was not able to speak. However, she was able to establish a comfortable relationship with her family and write down her problems and could read perfectly. She had a significant short height according to her age.

When she was 4 years old, she was diagnosed with type 1 DM, hypertension, hearing loss and related speech disorders, second-degree AV dissociation with intermittent ventricular extrasystoles, and cardiomegaly. She was also diagnosed with Hashimoto's thyroiditis at the age of 7 years in another hospital. She had no history of anemia during childhood.

Approximately 2 years ago, she was admitted to another hospital with severe anemia and hepatomegaly at the age of 30 years. Bone marrow biopsy showed hypercellularity according to her age, normal myeloid cells, erythroid hyperplasia, megaloblastic changes in premature erythroid cells, increased number of megakaryocytes, and few hypolobular, small, hyper-chromatic, mononuclear megakaryocytes. Sideroblasts were not seen with Prussian blue dye. Based on these findings, the patient was diagnosed with MDS. Darbepoetin was started at a dose of $100 \mu \mathrm{g} /$ week. The need for transfusion during this treatment continued. After 1 year of treatment, she came to the clinic, when she was 31 years old.

During the outpatient follow-ups, etiology of macrocytic anemia was re-investigated. Vitamin B12, folic acid, and thyroid hormone levels (with replacement therapy) were normal. Platelet count was also normal. Neutrophil count showed a slight decrease from time to time, although it did not decrease below $1,000 / \mathrm{mm}^{3}$. Because the need for transfusion persisted, darbepoetin dose was increased to $150 \mu \mathrm{g} /$ week and after 2 months, filgrastim was added at a dose of $30 \mathrm{lU} /$ week. 
During $12^{\text {th }}$ month of outpatient follow-up, she was hospitalised due to fatigue and abdominal distention. Her laboratory parameters were as follows: hemoglobin $(\mathrm{Hb}) 7.1 \mathrm{~g} / \mathrm{dl}$, mean corpuscular volume (MCV) 98/fL, platelets $173,000 / \mathrm{mm}^{3}$, leukocytes $7,300 / \mathrm{mm}^{3}$, neutrophils $5,060 / \mathrm{mm}^{3}$, lactate dehydrogenase (LDH) $297 \mathrm{U} / \mathrm{L}$, and creatinine $1.41 \mathrm{mg} / \mathrm{dl}$. No significant pathology was detected in hormonal and biochemical findings.

Upon cardiac examination, a systolic-diastolic murmur was detected. Cardiology consultation reported significant enlargement in the right voids, increased right ventricular trabeculation, third-degree tricuspid insufficiency, and left ventricular wall motion disturbance. Left ventricular ejection fraction was $48 \%$, and the pulmonary artery pressure was normal. Radiological evaluation of the patient with distension in the abdominal examination showed hepatomegaly, heart failure findings, and a large amount of free fluid in the abdomen. Optic atrophy was not observed in the patient, though she was using eyeglasses.

It was believed that she might have a different syndrome based on the early-onset hearing loss, type $1 \mathrm{DM}$, hypothyroidism, and cardiac problems. Her parents had a consanguineous marriage, and she had two healthy siblings. We explored the literature for clarifying the findings of our patient, which showed that these were consistent with TRMA. A genetic test was performed to confirm this syndrome, and $200 \mathrm{mg} /$ day of intramuscular thiamine was started. After 1 week, the dose was planned to be $100 \mathrm{mg} /$ day, and after 3 weeks, it was planned to be $100 \mathrm{mg} /$ week constantly. Darbepoetin and filgrastim treatments were stopped. Since the patient was symptomatic, erythrocyte replacement was performed. Treatment for type $1 \mathrm{DM}$ and hypothyroidism was also continued. The patient was discharged with the treatment recommendation and followed up weekly. First month after discharge, her $\mathrm{Hb}$ level was $10.5 \mathrm{~g} / \mathrm{dl}$ without erythrocyte transfusion. Genetic evaluation revealed a homozygous mutation in the SLC19A2 (603941) gene, which encodes a thiamine transporter protein, on chromosome 1q24. After three months of starting thiamine treatment, there was a slight improvement in her cardiac function on echocardiographic control (reduction in the right heart cavity and the tricuspid insufficiency). $\mathrm{Hb}$ level was $11.2 \mathrm{~g} / \mathrm{dl}$ in the third month of thiamine replacement therapy and $12 \mathrm{~g} / \mathrm{dl}$ in the fourth month. There was no significant reduction in the dose of insulin therapy. Levothyroxine replacement was continued. The patient is being followed up with weekly thiamine replacement therapy without further treatment for the anemia.

\section{DISCUSSION}

TRMA is inherited in an AR manner. Each sibling of an affected individual has a $25 \%$ TRMA syndrome risk. Therefore, prenatal tests in risk pregnancies may be useful in preventing disease. Typically, TRMA is diagnosed at an early age, usually by pediatricians. In the majority of cases reported in the literature, it has been observed that megaloblastic anemia is detected in early ages and helps in the early diagnosis of the syndrome.

The presence of megaloblastic anemia and ring sideroblasts in TRMA is mostly confused with MDS. However, MDS is a heterogeneous group of diseases usually seen in advanced ages and its treatment varies according to subgroups. There is a phenotypic similarity between TRMA and Wolfram syndrome (DIDMOAD: diabetes insipidus, diabetes mellitus, optic atrophy and deafness). Megaloblastic anemia and thiamine responsiveness are not observed in Wolfram syndrome.

Evaluation of the medical history and past reports of this patient showed that megaloblastic anemia appeared late, and therefore, the diagnosis of TRMA was made in the late period. To the author's knowledge, hypothyroidism has not been previously reported in patients with TRMA; thus, this case represents the first of its kind. We also believe that the regression of the cardiac dysfunction, which was evident in this case, at a mild level after the thiamine replacement therapy, is an important finding.

Wood and colleagues reported a 19-year male patient with TRMA syndrome who had myopia, astigmatism, and decreased peripheral visual fields, as well as megaloblastic anemia. ${ }^{8}$ The patient was also reported to have serious psychiatric problems. In contrast, this case is a female patient who was diagnosed at a later age. In addition, hypothyroidism and the presence of obvious cardiac problems in this case were not observed in the case reported by Wood and colleagues.

For patients with TRMA, administration of a thiamine replacement dose of $100 \mathrm{mg} /$ day appears to be sufficient, and dose adjustment can be performed according to clinical follow-up. ${ }^{9}$ However, in a pediatric case reported by Katipoglu and colleagues, $100 \mathrm{mg} /$ day treatment for megaloblastic anemia was initially adequate, but there was a significant decrease in the $\mathrm{Hb}$ of the patient in the fourth-year follow-up. After increasing the thiamine dose to $200 \mathrm{mg} / \mathrm{day}$, the $\mathrm{Hb}$ level was normalised again. 10 In this case, thiamine replacement was initially given at a high dose of 200 $\mathrm{mg} / \mathrm{day}$. The dose was reduced and planned to be 100 $\mathrm{mg} /$ week after one month. With this dose, $\mathrm{Hb}$ reached normal levels at the fourth month. It was planned that the dose may be increased, if necessary, according to the patient's regular follow-up.

Distinguishing TRMA from MDS can sometimes be difficult. In the diagnosis of adult megaloblastic anemia, all accompanying clinical tables and hereditary and acquired etiologies should be evaluated. We believe that 
by identifying genetic diseases, such as TRMA, at an early age, the patients can be prevented from various irreversible complications.

\section{REFERENCES}

1. Porter FS, Rogers LE, Sidbury JB Jr. Thiamine-responsive megaloblastic anemia. J Pediatr 1969; 74:494-504.

2. Meire FM, Van Genderen MM, Lemmens K, Ens-Dokkum MH Thiamine-responsive megaloblastic anaemia syndrome (TRMA) with cone-rod dystrophy. Ophthalmic Genet 2000; 21:243-50.

3. Lorber A, Gazit AZ, Khoury A, Schwartz Y, Mandel H. Cardiac manifestations in thiamine-responsive megaloblastic anemia syndrome. Pediatr Cardiol 2003; 24:476-81.

4. Fleming JC, Tartaglini E, Steinkamp MP, Schorderet DF, Cohen N, Neufeld EJ. The gene mutated in thiamine-responsive anaemia with diabetes and deafness (TRMA) encodes a functional thiamine transporter. Nat Genet 1999; 22:305-8.

5. Oishi K, Diaz GA. Thiamine-responsive megaloblastic anemia syndrome. GeneReviews $\AA$ [Internet]. Seattle (WA): University of Washington, Seattle; 1993-2018. 2003 Oct 24 [updated 2017 May 4].
6. Boros LG, Steinkamp MP, Fleming JC, Lee WN, Cascante M, Neufeld EJ. Defective RNA ribose synthesis in fibroblasts from patients with thiamine-responsive megaloblastic anemia (TRMA). Blood 2003; 102:3556-61.

7. Fleming JC, Steinkamp MP, Kawatsuji R, Tartaglini E, Pinkus JL, Pinkus GS, et al. Characterization of a murine high-affinity thiamine transporter, SLC19A2. Mol Genet Metab 2001; 74: 273-80.

8. Wood MC, Tsiouris JA, Velinov M. Recurrent psychiatric manifestations in thiamine-responsive megaloblastic anemia syndrome due to a novel mutation c.63_71 delACCGCTC in the gene SLC19A2. Psychiatry Clin Neurosci 2014; 68:487.

9. Mikstiene V, Songailiene J, Byckova J, Rutkauskiene G, Jasinskiene $\mathrm{E}$, Verkauskiene $\mathrm{R}$, et al. Thiamine responsive megaloblastic anemia syndrome: A novel homozygous SLC19A2 genemutation identified. Am J Med Genet A 2015; 167:1605-9.

10. Katipoglu N, Karapinar TH, Demir K, Aydin Köker S, Nalbantoglu Ö, Ay Y, et al. Infantile-onset thiamine responsive megaloblastic anemia syndrome with SLC19A2 mutation: A case report. Arch Argent Pediatr 2017; 115:e153-6. 